OR Spektrum (1991) 13:15-30

\title{
Coincidence of and collinearity between game theoretic solutions
}

\author{
T. S. H. Driessen ${ }^{1}$ and Y. Funaki ${ }^{2}$ \\ 1 Faculty of Applied Mathematics, University of Twente, Enschede, The Netherlands \\ ${ }^{2}$ Faculty of Economics, Toyo University, Hakusan, Bunkyo-ku, Tokyo 112, Japan
}

Received July 27, 1989 / Accepted June 1, 1990

Summary. The first part is the study of several conditions which are sufficient for the coincidence of the prenucleolus concept and the egalitarian nonseparable contribution (ENSC-) method. The main sufficient condition for the coincidence involved requires that the maximal excesses at the ENSC-solution are determined by the $(n-1)$-person coalitions in the $n$-person game. The second part is the study of both a new type of games, the so-called $k$ coalitional $n$-person games, and the interrelationship between solutions on the class of those games. The main results state that the Shapley value of a $k$-coalitional $n$ person game can be written as a convex or affine combination of the ENSC-solution and the centre of the imputation set.

Zusammenfassung. Im ersten Teil der Arbeit werden verschiedene hinreichende Bedingungen für die Koinzidenz des Prenukleolus-Lösungskonzepts und der ENSCRegel vorgestellt. Es wird dabei gezeigt, daß der Prenukleolus mit der ENSC-Lösung zusammenfällt, falls die maximalen Exzesse der ENSC-Lösung durch die $(n-1)$ Personen Koalitionen des $n$-Personenspiels bestimmt werden. Im zweiten Teil der Arbeit untersuchen wir eine Klasse von Spielen, die sogenannten $k$-Koalitions- $n$-Personenspiele und untersuchen die Zusammenhänge zwischen den Lösungskonzepten für diesen speziellen Typ von Spielen. Es stellt sich heraus, daß der Shapleywert eines $k$-Koalitions- $n$-Personenspiels beschrieben werden kann als eine Linearkombination der ENSC-Lösung und des Schwerpunktes der Auszahlungsmenge.

\section{Introduction}

There are many solution concepts for cooperative games in characteristic function form. The existence of relationships between solution concepts is an important issue for the theory of cooperative games as well as the application of game theoretic analysis to real problems (e.g., the cost allocation problem).
Generally speaking, relationships between the distinct solution concepts are known to some extent. For instance, the bargaining set includes both the kernel and the core, while the intersection of the kernel with the nonempty core contains the nucleolus. The Shapley value of any convex game coincides with the centre of gravity of the core, whereas the Shapley value of an arbitrary balanced game may fall outside the core.

In the context of game theoretic applications, the onepoint solution concepts of the (pre)-nucleolus and the Shapley value are widely used. Further, the separable contributions remaining benefits (SCRB)-method is a well-known cost allocation method in the water resources field. The main goal of the paper is to establish relationships between the (pre)-nucleolus, the Shapley value and the egalitarian nonseparable contribution (ENSC-) method which is a simple variant of the SCRB-method. The ENSC-solution is based on the separable contributions which are one of the various marginal contributions that are taken into account for the determination of the Shapley value. In addition, the equal excesses of the $(n-1)$-person coalitions at the ENSC-solution of any $n$ person game give rise to compare the ENSC-method with the (pre)-nucleolus concept which is defined in terms of excesses.

The organization of the paper is as follows. Section 2 treats the relevant game theoretic notions and solution concepts, e.g., the (pre)-nucleolus. In Sect. 3 we present the ENSC-method and we review several sufficient conditions for the coincidence of the ENSC-solution and the prenucleolus. The main sufficient condition (3.1) requires the effectiveness of the $(n-1)$-person coalitions with respect to the excesses at the ENSC-solution.

In Sect. 4 we study the set of pre-imputations for which the $(n-1)$-person coalitions are effective with respect to the excesses. Due to a close determination of the intersection of the set involved with the prekernel (cf. Theorem 4.6), we formulate a sufficient condition for the coincidence of the prenucleolus and the ENSC-solution. The main Theorem 4.7 states that both solutions coincide whenever the ENSC-solution belongs to the set involved. 
Section 5 deals with one specific type of games, the socalled glove games. The coincidence of the (pre)-nucleolus concept and the ENSC-method on the class of glove games is established with the aid of the results obtained in Sect. 3. The main Theorem 5.2 expresses that the Shapley value of any glove game is a convex combination of the ENSC-solution and the centre of the imputation set. In Sect. 6 we introduce the class of $k$-coalitional $n$-person games and describe the locus of the Shapley value for this type of games. The main Theorems 6.3,6.6 and 6.9 state that the Shapley value of a $k$-coalitional game can be written as a convex or affine combination of the ENSCsolution and the centre of the imputation set. Section 6 consists of three subsections which correspond to the cases $k=n-1, k=1$ and $2 \leq k \leq n-2$ respectively. In the final Section 7 we list two concluding remarks.

\section{Notions and solution concepts}

A cooperative n-person game in characteristic function form is an ordered pair $(N ; v)$, where the player set $N=\{1,2, \ldots, n\}, n \geq 3$, and the characteristic function $v: 2^{N} \rightarrow \mathbb{R}$ is a real-valued set-function on the set $2^{N}$ of all subsets of $N$. The worth $v(S)$ of coalition $S \subset N$ in the game $v$ represents the profits obtained solely by cooperation between the members of $S$. The number of players in a coalition $S$ is denoted by $|S|$. It is always required that the worth of the empty coalition is zero, i.e., $v(\varnothing):=0$. The class of all cooperative $n$-person games is denoted by $G^{n}$.

A distribution of the total worth $v(N)$ among the $n$ players is represented by an $n$-tuple $x=\left(x_{1}, x_{2}, \ldots, x_{n}\right) \in \mathbb{R}^{n}$ of real numbers satisfying the efficiency principle $\sum_{j \in N} x_{j}=v(N)$. In case the payoff $x_{i}$ to any player $i \in N$ is at least his own worth in the game $v$, i.e., $x_{i} \geq v(\{i\})$ for all $i \in N$, then we say the efficient payoff vector $x \in \mathbb{R}^{n}$ meets the individual rationality principle. Besides, the group rationality principle may be met by the efficient payoff vector. In accordance with these principles, we define the pre-imputation set $I^{*}(v)$, the imputation set $I(v)$ and the core $C(v)$ of the $n$-person game $v$ by

$I^{*}(v):=\left\{x \in \mathbb{R}^{n} \mid x(N)=v(N)\right\}$

$I(v) \quad:=\left\{x \in I^{*}(v) \mid x_{i} \geq v(\{i\}) \quad\right.$ for all $\left.i \in N\right\}$

$C(v):=\left\{x \in I^{*}(v) \mid x(S) \geq v(S) \quad\right.$ for all $\left.S \subset N\right\}$,

where $x(S):=\sum_{j \in S} x_{j}$ for all $S \subset N, S \neq \varnothing$, and $x(\varnothing):=0$.

In other words, the core consists of pre-imputations that give rise only to nonpositive excesses. Here the excess of coalition $S$ with respect to the pre-imputation $x$ in the game $v$ is defined to be

$\mathrm{e}^{v}(S, x):=v(S)-x(S)=v(S)-\sum_{j \in S} x_{j}$.
The idea of excess forms the basis of several solution concepts for cooperative games such as the core, the (pre)nucleolus and the prekernel. The definition of the (pre)nucleolus of an $n$-person game $v$ is as follows. We associate with any pre-imputation $x \in I^{*}(v)$ the complaint vector $\Theta(x) \in \mathbb{R}^{2^{n}}$ whose components are the excesses $\mathrm{e}^{v}(S, x)$, $S \subset N$, arranged in nonincreasing order. The (pre)-nucleolus consists of (pre)-imputations that minimize the complaint function $\Theta(x)$ in the lexicographic order $\leq_{L}$ on $\mathbb{R}^{2^{n}}$ over the (pre)-imputation set. Thus,

$\mathcal{N}^{*}(v):=\left\{x \in I^{*}(v) \mid \Theta(x) \leq_{L} \Theta(y) \quad\right.$ for all $\left.y \in I^{*}(v)\right\}$

and

$\mathcal{N}(v):=\left\{x \in I(v) \mid \Theta(x) \leq_{L} \Theta(y) \quad\right.$ for all $\left.y \in I(v)\right\}$.

The prekernel of the game $v$ is defined to be

$\mathscr{K}^{*}(v):=\left\{x \in I^{*}(v) \mid s_{i j}^{v}(x)=s_{j i}^{v}(x) \quad\right.$ for all $\left.i, j \in N, i \neq j\right\}$

where the maximum surplus of player $i$ over another player $j$ with respect to the pre-imputation $x$ in the game $v$ is given by

$s_{i j}^{v}(x):=\max \left[e^{v}(S, x) \mid S \subset N, i \in S, j \notin S\right]$.

It is well-known that the (pre)-nucleolus is a singleton and the prenucleolus is included in the prekernel (cf. Schmeidler 1969). The unique point in the nucleolus and the prenucleolus respectively are denoted by $\eta(v)$ and $\eta^{*}(v)$. Clearly, the prenucleolus of a game coincides with the nucleolus of the game whenever the prenucleolus is individually rational. Further, the nucleolus is always contained in a nonempty core. In the numerical examples we delete the braces for the sake of notation and we write $i$ instead of the set $\{i\}$, etc.

\section{Coincidence of the prenucleolus and the egalitarian nonseparable contribution method}

A standard principle requires that the payoff to any player $i \in N$ in the game $v$ is at most the separable contribution $S C_{i}(v)=v(N)-v(N-\{i\})$ of player $i$ with respect to the formation of the grand coalition $N$. The egalitarian nonseparable contribution (ENSC-) method is characterized by the fact that the remaining nonseparable contribution $\mathrm{NSC}(v)=v(N)-\sum_{j \in N} S C_{j}(v)$ is equally charged to the $n$ players in the game $v$. That is

$\operatorname{ENSC}_{i}(v):=S C_{i}(v)+n^{-1} \mathrm{NSC}(v) \quad$ for all $i \in N$.

As such, the ENSC-method can be regarded as a naive version of the well-known separable contributions remaining benefits (SCRB-) method which is a widely used approach in the water resources field (cf. Young, et al. 1982).

The purpose of the section is to review one main and several related conditions which are sufficient for the 
coincidence of the ENSC-method and the prenucleolus concept. The idea of the main condition involved was mentioned for the first time in Suzuki and Nakayama (1976). They require that the maximal excesses at the ENSC-solution are determined by the $(n-1)$-person coalitions, i.e.,

$e^{v}(S, z) \leq e^{v}(N-\{i\}, z)$

for all $i \in N$ and all $S \subset N-\{i\}, S \neq \varnothing$, where $z=$ $\operatorname{ENSC}(v)$.

In the context of excess, we say that the $(n-1)$-person coalitions are effective at the ENSC-solution. For the sake of completeness, we also present a proof of the following main theorem.

Theorem 3.1. Let $v \in G^{n}$ and put $z:=\operatorname{ENSC}(v)$. Then $\eta^{*}(v)=z$ whenever the effectiveness condition (3.1) for $z$ holds.

Proof. Put $x:=\eta^{*}(v)$. Suppose that the effectiveness condition (3.1) for $z$ holds. Obviously, we have for all $i \in N$

$$
\begin{aligned}
e^{v}(N-\{i\}, z) & =v(N-\{i\})-z(N-\{i\}) \\
& =z_{i}-S C_{i}(v)=n^{-1} \mathrm{NSC}(v)
\end{aligned}
$$

Together with (3.1), this yields

$e^{v}(S, z) \leq e^{v}(N-\{i\}, z)=n^{-1} \mathrm{NSC}(v)$

for all $i \in N$ and all $S \subset N-\{i\}, S \neq \varnothing$.

In the lexicographic comparison between the two complaint vectors $\Theta(x)$ and $\Theta(z)$, we may ignore the excesses of $N$ and $\varnothing$ since $e^{v}(S, x)=e^{v}(S, z)=0$ whenever $S=N$ or $S=\varnothing$. Therefore, we obtain $\Theta_{1}(z)=n^{-1} \mathrm{NSC}(v)$. Furthermore, we have $\Theta(x) \leq_{L} \Theta(z)$ because of $x=\eta^{*}(v)$. Now it follows that for all $i \in N$

$x_{i}-S C_{i}(v)=v(N-\{i\})-x(N-\{i\})=e^{v}(N-\{i\}, x)$

$\leq \Theta_{1}(x) \leq \Theta_{1}(z)=n^{-1} \mathrm{NSC}(v)$.

So, $x_{i} \leq S C_{i}(v)+n^{-1} \mathrm{NSC}(v)=z_{i}$ for all $i \in N$. From $x \leq z$ and $x(N)=v(N)=z(N)$, we conclude the vector equality $x=z$. Thus, $\eta^{*}(v)=z$ as was to be shown.

In the remainder of the section we pay attention to conditions which are stronger than the effectiveness condition (3.1). In order to present a uniform treatment of the several related conditions, we introduce the notion of the gap function $g^{\nu}: 2^{N} \rightarrow \mathbb{R}$ corresponding to the characteristic function $v: 2^{N} \rightarrow \mathbb{R}$. The gap $g^{v}(S)=\sum_{j \in S} S C_{j}(v)$ $-v(S)$ of coalition $S$ in the game $v$ represents the loss (or gain) to the coalition $S$ whenever its worth is compared with the total amount of the separable contributions of its members. By straightforward calculations, we obtain $g^{v}(N)=-\mathrm{NSC}(v), g^{v}(N-\{i\})=g^{v}(N)$ for all $i \in N$, and in addition, $C(v)=\varnothing$ whenever there exists a coalition $S \subset N$ with $g^{v}(S)<0$. Consequently, the nonnegativity of the gap function $g^{v}$ is a necessary condition for the nonemptiness of the core of the game $v$.

In the following three subsections we first review the conditions, as mentioned in Funaki (1986), Legros (1986) and Driessen $(1983 ; 1985 ; 1988$, page 73$)$ respectively, and secondly, we reformulate their conditions in terms of the gap function.

Subsection 3.1. Funaki's approach to the study of the ENSC-method is based on the condition that determines whether or not the ENSC-solution is bounded above by the smallest contributions of the single players to the nonempty coalitions. In terms of the game $v$ itself, Funaki's condition is specified as follows:

$S C_{i}(v)+n^{-1} \mathrm{NSC}(v) \leq v(S \cup\{i\})-v(S)$

for all $i \in N$ and all $S \subset N-\{i\}, S \neq \varnothing$.

The equivalent condition in terms of the corresponding gap function $g^{v}$ is given by

$g^{v}(S \cup\{i\})-g^{v}(S) \leq n^{-1} g^{v}(N)$

for all $i \in N$ and all $S \subset N-\{i\}, S \neq \varnothing$.

Subsection 3.2. Legros' approach to the study of the ENSC-method is based on the so-called pseudo-convexity condition. Opposite to the condition (3.2), pseudo-convexity requires that all the contributions of the single players are bounded above by their separable contributions. In terms of the game $v$ itself, Legros' pseudoconvexity condition is specified as follows:

$v(S \cup\{i\})-v(S) \leq S C_{i}(v)$,

$S C_{i}(v)-v(\{i\}) \geq-n^{-1}(n-1) \mathrm{NSC}(v)$

for all $i \in N$ and all $S \subset N-\{i\}$.

The equivalent condition in terms of the corresponding gap function $g^{v}$ is given by

$g^{v}(S \cup\{i\}) \geq g^{v}(S)$,

$g^{v}(\{i\}) \geq n^{-1}(n-1) g^{v}(N)$

for all $i \in N$ and all $S \subset N-\{i\}$.

The condition (3.5) is known as the monotonicity condition for the gap function $g^{v}$ and as a consequence, the gap of the grand coalition is at the top level. For any game with many players, the two conditions (3.5)-(3.6) imply that the corresponding gap function is almost constant.

Subsection 3.3. Driessen's approach to the study of the ENSC-method is based on the so-called 1-convexity condition. Here 1-convexity requires that the contribution of any coalition with respect to the formation of the grand 
coalition $N$ is at least the total amount of the separable contributions of the members of the coalition involved. In terms of the game $v$ itself, Driessen's 1-convexity condition is specified as follows:

$\mathrm{NSC}(v) \leq 0 \quad$ and $\quad v(N)-v(N-S) \geq \sum_{j \in S} S C_{j}(v)$
for all $S \subset N, S \neq N$.

The equivalent condition in terms of the corresponding gap function $g^{v}$ expresses that the nonnegative gap of the grand coalition is at the bottom level, i.e.,

$0 \leq g^{v}(N) \leq g^{v}(S)$ for all $S \subset N, S \neq \varnothing$.

Subsection 3.4. The relationship between the effectiveness condition (3.1) and the other conditions will be derived from the next characterization of the effectiveness condition (3.1) in terms of the gap function.

Proposition 3.2. Let $v \in G^{n}$ and put $z:=\operatorname{ENSC}(v)$.

(i) The effectiveness condition (3.1) for $z$ is equivalent to

$n^{-1} g^{v}(N) \leq(|S|+1)^{-1} g^{v}(S) \quad$ for all $S \subset N, S \neq N, \varnothing$.

(ii) If (3.8) holds and $g^{v}(N) \geq 0$, then $\eta(v)=\eta^{*}(v)=$ $\operatorname{ENSC}(v) \in C(v)$.

Proof. (i) For any coalition $S$, the excess of $S$ at $z$ satisfies

$$
\begin{aligned}
\mathrm{e}^{\mathrm{v}}(S, z) & =v(S)-\sum_{j \in S} S C_{j}(v)-n^{-1}|S| \mathrm{NSC}(v) \\
& =-g^{v}(S)+n^{-1}|S| g^{v}(N)
\end{aligned}
$$

In particular, we get that $\mathrm{e}^{\mathrm{v}}(N-\{i\}, z)=-n^{-1} g^{v}(N)$ for all $i \in N$. Now it follows that the effectiveness condition (3.1) for $z$ is equivalent to

$\mathrm{e}^{v}(S, z) \leq-n^{-1} g^{v}(N) \quad$ for all $S \subset N, S \neq N, \varnothing$

or equivalently,

$-g^{v}(S)+n^{-1}|S| g^{v}(N) \leq-n^{-1} g^{v}(N)$

for all $S \subset N, S \neq N, \varnothing$.

From this we conclude that the equivalence $(3.1) \Leftrightarrow(3.8)$ holds.

(ii) Suppose that (3.8) holds and $g^{v}(N) \geq 0$. Because (3.8) is equivalent to (3.9), it follows that $\mathrm{e}^{v}(S, z) \leq 0$ for all $S \subset N, S \neq N, \varnothing$. Hence, $z \in C(v)$. The equalities $z=\eta^{*}(v)=\eta(v)$ are due to Theorem 3.1 and the individual rationality of the prenucleolus respectively.

Theorem 3.3. Let $v \in G^{n}$. If Funaki's condition (3.3), Legros' condition (3.5)-(3.6) or Driessen's condition (3.7) holds, then the effectiveness condition (3.1) holds and
$\eta(v)=\eta^{*}(v)=\operatorname{ENSC}(v) \in C(v)$.

Proof. Suppose that (3.3), (3.5)-(3.6) or (3.7) holds. In view of Proposition 3.2, it is sufficient to show $g^{v}(N) \geq 0$ and

$-g^{v}(S)+n^{-1}|S| g^{v}(N) \leq-n^{-1} g^{v}(N)$

for all $S \subset N, S \neq N, \varnothing$.

The equality holds whenever $|S|=n-1$. Thus, let $S \subset N$ be such that $1 \leq|S| \leq n-2$. We distinguish three cases.

Case one. Suppose that (3.7) holds. Then we have $0 \leq g^{v}(N) \leq g^{v}(S)$ and hence,

$$
\begin{aligned}
-g^{v}(S) & +n^{-1}|S| g^{v}(N) \leq-g^{v}(N) \\
& +n^{-1}(n-1) g^{v}(N)=-n^{-1} g^{v}(N) .
\end{aligned}
$$

Case two. Suppose that (3.5)-(3.6) holds. Take $i \in S$. By the monotonicity condition (3.5) and (3.6), we obtain $g^{v}(N) \geq g^{v}(\varnothing)=0$ as well as $g^{v}(S) \geq g^{v}(\{i\}) \geq$ $n^{-1}(n-1) g^{v}(N)$. Now it follows that

$$
\begin{aligned}
-g^{v}(S)+n^{-1}|S| g^{v}(N) & \leq-n^{-1}(n-|S|-1) g^{v}(N) \\
& \leq-n^{-1} g^{v}(N) .
\end{aligned}
$$

Case three. Suppose that (3.3) holds. Take $i \in N-S$. From (3.3) we derive $n^{-1} g^{v}(N) \geq g^{v}(N)-g^{v}(N-\{i\})=0$ and so, $g^{v}(N) \geq 0$. Further, we obtain $g^{v}(N-\{i\})-g^{v}(S)$ $\leq n^{-1}(n-|S|-1) g^{v}(N)$ by applying repeatedly the condition (3.3). Together with $g^{v}(N-\{i\})=g^{v}(N)$, this yields

$$
\begin{aligned}
-g^{v}(S) & +n^{-1}|S| g^{v}(N) \leq-g^{v}(N-\{i\}) \\
& +n^{-1}(n-1) g^{v}(N)=-n^{-1} g^{\nu}(N) .
\end{aligned}
$$

Thus, (3.8) holds and $g^{v}(N) \geq 0$. This completes the proof.

For games $v$ satisfying (3.8) and $g^{v}(N) \geq 0$, the core is nonempty, but the locus of the ENSC-solution inside the core may be arbitrary. However, 1-convex games possess a very regular core structure in such a way that the ENSCsolution is the centre of gravity of the vertices of the core (cf. Driessen 1983, 1985, 1988). Further, the intersection of the (pre)-kernel with the core of a 1-convex game is a singleton consisting of the ENSC-solution. Finally, the theory of this section is illustrated by a practical example concerning Japan.

Example 3.4. We consider the model of the cooperative water resource development as described in Suzuki and Nakayama (1976). Especially, we pay attention to their model applied to the case of Kanagawa prefecture in Japan. There are two agricultural associations (of the Rivers Sakawa and Sagami, denoted as 1 and 2) who view the existing water supplies as being more than adequate for their own irrigation needs, and three city water service authorities (of the cities Kanagawa, Yokohama and 
Table 1

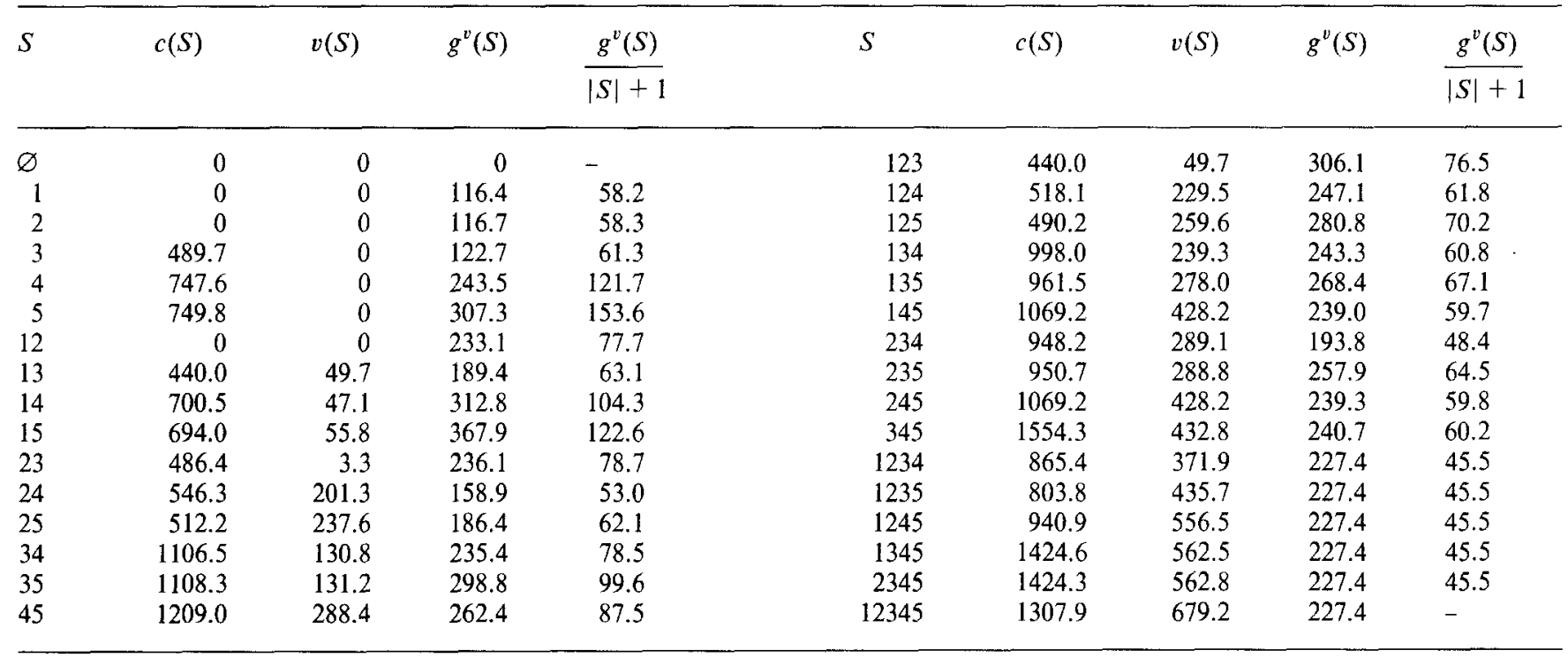

Kawasaki, denoted as 3,4 and 5) whose current or future water needs are not met by existing sources. Each city might acquire the quantity of additional water it needs in two ways: construct a dam on the River Sakawa with or without the cooperation of other cities and/or arrange with the agricultural associations for the direct diversion of water from them to the city. The characteristic cost function $c$ is derived from the minimum cost $c(S)$ of meeting the additional water needs of the cities in $S$, on the understanding that there is no cooperation from those agents outside $S$.

The data concerning the cost figures in $10^{8}$ yen is taken from Suzuki and Nakayama (1976, page 1085) and is listed in Table 1 . With the cost function $c: 2^{N} \rightarrow \mathbb{R}$ we associate the cost savings function $v: 2^{N} \rightarrow \mathbb{R}$ by means of $v(S):=\sum_{j \in S} c(\{j\})-c(S)$ for all $S \subset N$. The corresponding saving figures in $10^{8}$ yen is also listed in Table 1. In the same table we observe that the associated savings game $v$ satisfies neither of the three conditions (3.3), (3.5)(3.6), (3.7), but nevertheless, the condition (3.8) is satisfied by the game $v$. Therefore, we obtain the coincidence $\eta^{*}(v)=\operatorname{ENSC}(v)$ and as such, the prenucleolus cost allocation is determined by

$\eta_{i}^{*}(c)=c(\{i\})-\eta_{i}^{*}(v)=c(\{i\})-\operatorname{ENSC}_{i}(v)$ for all $i \in N$.

In view of this, straightforward calculations yield

$\eta^{*}(c)=(-70.92,-71.22,412.48,549.58,487.98)$.

\section{Properties of the prekernel and the prenucleolus}

In addition to his study of the ENSC-method, Funaki (1986) investigated the behaviour of both the prekernel and the prenucleolus with respect to the smallest contributions of the single players to the nonempty coalitions. Funaki's main result states that those smallest contribu- tions give rise to an upper bound for the prenucleolus if and only if the ENSC-solution is bounded above by those smallest contributions, i.e., iff the condition (3.2) holds. In this section we derive a similar result for the case that the relevant notion of the smallest contribution of any player to nonempty coalitions is changed into the smallest contribution of any coalition with respect to the formation of $(n-1)$-person coalitions.

Let $v \in G^{n}$. The smallest contribution of coalition $S$ with respect to the formation of $(n-1)$-person coalitions in the $n$-person game $v$ is defined to be

$$
\begin{aligned}
m^{v}(S):= & \min [v(N-\{j\})-v((N-\{j\})-S) \mid j \in N-S] \\
& \text { for all } S \subset N, S \neq N,
\end{aligned}
$$

$m^{v}(N):=v(N)$

Remark that $m^{v}(\varnothing)=0$ and $m^{v}(N-\{i\})=v(N-\{i\})$ for all $i \in N$. According to the next proposition, the smallest contributions of the coalitions with respect to the formation of $(n-1)$-person coalitions are useful to characterize the effectiveness condition (4.2) for an arbitrary payoff vector $x \in \mathbb{R}^{n}$.

Proposition 4.1. Let $v \in G^{n}$ and $x \in \mathbb{R}^{n}$. The following statements are equivalent.

(i) $\mathrm{e}^{v}(S, x) \leq \mathrm{e}^{v}(N-\{i\}, x)$

for all $i \in N$ and all $S \subset N-\{i\}, S \neq \varnothing$

(ii) $x(S) \leq m^{v}(S)$ for all $S \subset N$ with $1 \leq|S| \leq n-2$

Proof. Evidently, we have the following equivalences:

$x(S) \leq m^{v}(S) \quad$ for all $S \subset N$ with $1 \leq|S| \leq n-2$

$\Leftrightarrow x(S) \leq v(N-\{j\})-v((N-\{j\})-S)$ 
for all $S \subset N$ with $1 \leq|S| \leq n-2$, for all $j \in N-S$

$\Leftrightarrow \mathrm{e}^{v}((N-\{j\})-S, x) \leq \mathrm{e}^{v}(N-\{j\}, x)$

for all $S \subset N$ with $1 \leq|S| \leq n-2$, for all $j \in N-S$

$\Leftrightarrow \mathrm{e}^{v}(T, x) \leq \mathrm{e}^{v}(N-\{j\}, x)$

for all $j \in N$ and all $T \subset N-\{j\}, T \neq \varnothing$.

So, the equivalence $(4.2) \Leftrightarrow(4.3)$ holds.

In view of the condition (4.3), we define the set $U(v) \subset \mathbb{R}^{n}$ by

$$
\begin{aligned}
U(v):= & \left\{x \in I^{*}(v) \mid x(S) \leq m^{v}(S) \text { for all } S \subset N\right. \\
& \text { with } 1 \leq|S| \leq n-2\} .
\end{aligned}
$$

Thus, the set $U(v)$ consists of pre-imputations that give rise only to payoffs not greater than the relevant smallest contributions for all coalitions containing at most $n-2$ players. Proposition 4.1 expresses that the effectiveness condition (4.2) for the efficient payoff vector $x \in I^{*}(v)$ is satisfied if and only if $x \in U(v)$. Due to Theorem 3.1, we obtain the coincidence $\eta^{*}(v)=\operatorname{ENSC}(v)$ whenever $\operatorname{ENSC}(v) \in U(v)$.

Clearly, the core of the game $v$ can be written as

$$
C(v)=\left\{x \in I^{*}(v) \mid x(S) \leq v(N)-v(N-S) \text { for all } S \subset N\right\} .
$$

In other words, the payoffs according to any core-element are bounded above by the contributions of the coalitions with respect to the formation of the grand coalition $N$. Analogously, the payoffs according to any element of the set $U(v)$ are bounded above by the smallest contributions of the coalitions with respect to the formation of $(n-1)$ person coalitions. In the remainder of the section we compare the location of the set $U(v)$ with the core $C(v)$ and the prekernel $\mathscr{K}^{*}(v)$. We first treat two examples.

Example 4.2. Consider the 3-person game $v$ as listed in the second row.

\begin{tabular}{lcccccrr}
$S$ & $\{1\}$ & $\{2\}$ & $\{3\}$ & $\{1,2\}$ & $\{1,3\}$ & $\{2,3\}$ & $N$ \\
\hline$v(S)$ & 0 & 0 & 0 & 9 & 9 & 15 & 18 \\
$g^{v}(S)$ & 3 & 9 & 9 & 3 & 3 & 3 & 3 \\
$m^{v}(S)$ & 9 & 9 & 9 & 9 & 9 & 15 & 18
\end{tabular}

Then the separable contributions are given by $S C_{i}(v)=3,9,9$ for $i=1,2,3$ respectively. The corresponding gap function $g^{v}$ is listed in the third row, from which we derive that the game $v$ satisfies Driessen's 1convexity condition (3.7) as well as Funaki's condition (3.3), whereas Legros' pseudo-convexity condition (3.5)(3.6) does not hold. By applying Theorem 3.3, we obtain the coincidence $\eta^{*}(v)=\operatorname{ENSC}(v)=(2,8,8)$. Due to the $1-$ convexity of the game $v$, the core is the triangle with the three vertices $(0,9,9),(3,6,9)$ and $(3,9,6)$. Note that the ENSC-solution is the centre of gravity of the three vertices of the core. The function $m^{v}$ of (4.1) is listed in the fourth row, from which we deduce that the set $U(v)$ is given by
$U(v)=\left\{x \in \mathbb{R}^{3} \mid x_{1}+x_{2}+x_{3}=18, x_{i} \leq 9\right.$ for all $\left.i \in N\right\}$.

Thus, the set $U(v)$ is the convex hull of the three points $(0,9,9),(9,0,9)$ and $(9,9,0)$. We conclude that the inclusion $C(v) \subset U(v)$ is strict and furthermore, $\operatorname{ENSC}(v) \in U(v)$.

Example 4.3. Consider the 3-person game $w$ as listed in the second row.

\begin{tabular}{lccccccc}
$S$ & $\{1\}$ & $\{2\}$ & $\{3\}$ & $\{1,2\}$ & $\{1,3\}$ & $\{2,3\}$ & $N$ \\
\hline$w(S)$ & 0 & 0 & 0 & 10 & 11 & 12 & 15 \\
$g^{w}(S)$ & 3 & 4 & 5 & -3 & -3 & -3 & -3 \\
$\frac{g^{w}(S)}{|S|+1}$ & 1.5 & 2 & 2.5 & -1 & -1 & -1 & - \\
$m^{w}(S)$ & 10 & 10 & 11 & 10 & 11 & 12 & 15
\end{tabular}

Then $S C_{i}(w)=3,4,5$ for $i=1,2,3$ respectively. From $g^{w}(N)=-3<0$, it follows immediately that the core is empty and none of the three conditions (3.3), (3.5)-(3.6), (3.7) does hold. Nevertheless, from the fourth row we derive that the game $w$ satisfies the main condition (3.8) and hence, we obtain the coincidence $\eta^{*}(w)=$ $\operatorname{ENSC}(w)=(4,5,6)$. Notice that $\operatorname{ENSC}(w) \in U(w)$ because the set $U(w)$ is given by

$$
\begin{aligned}
U(w)= & \left\{x \in \mathbb{R}^{3} \mid x_{1}+x_{2}+x_{3}=15, x_{1} \leq 10, x_{2} \leq 10,\right. \\
& \left.x_{3} \leq 11,\right\} .
\end{aligned}
$$

From the first example we learn that the set $U(v)$ may contain points outside the core $C(v)$. The next theorem, however, states that the part of the set $U(v)$ inside the core can be characterized as the intersection of the set $U(v)$ with the comprehensive orthant $O(v)$ that is bounded above by the separable contributions. As a consequence, a necessary and sufficient condition for the inclusion $U(v) \subset C(v)$ can be given in terms of the boundedness of the set $U(v)$ by the separable contributions.

Theorem 4.4. Let $v \in G^{n}$ and put $O(v):=\left\{x \in \mathbb{R}^{n} \mid x_{i} \leq\right.$ $S C_{i}(v)$ for all $\left.i \in N\right\}$.

(i) $U(v) \cap C(v)=U(v) \cap O(v)$

(ii) $U(v) \subset C(v)$ iff $U(v) \subset O(v)$.

Proof. (i) Note that the inclusion $C(v) \subset O(v)$ holds since

$x_{i}=v(N)-x(N-\{i\}) \leq v(N)-v(N-\{i\})=S C_{i}(v)$

for all $x \in C(v)$ and all $i \in N$. So, it remains to establish the inclusion $U(v) \cap O(v) \subset U(v) \cap C(v)$. Let $x \in U(v) \cap O(v)$ and $S \subset N, S \neq N, \varnothing$. We show $x(S) \geq v(S)$ or equivalently, $\mathrm{e}^{v}(S, x) \leq 0$. Because $S \neq N$, there exists $i \in N$ such that $S \subset N-\{i\}$. By Proposition 4.1, we deduce from $x \in U(v)$ that

$$
\begin{aligned}
\mathrm{e}^{v}(S, x) \leq \mathrm{e}^{v}(N-\{i\}, x) & =v(N-\{i\})-x(N-\{i\}) \\
& =x_{i}-S C_{i}(v) \leq 0
\end{aligned}
$$


where the last inequality follows from $x \in O(v)$. Therefore, $x \in C(v)$ as was to be shown.

(ii) The equivalence is a direct consequence of part (i).

The following results concerning the intersection of the set $U(v)$ with the prekernel are established with the aid of the lemma which gives a characterization of the effectiveness condition (4.2) in terms of the maximum surplus. The proof of the lemma is straightforward and is left to the reader.

Lemma 4.5. Let $v \in G^{n}$ and $x \in \mathbb{R}^{n}$. The following statements are equivalent.

(i) $\mathrm{e}^{v}(S, x) \leq \mathrm{e}^{v}(N-\{i\}, x)$

for all $i \in N$ and all $S \subset N-\{i\}, S \neq \varnothing$

(ii) $s_{i j}^{v}(x)=\mathrm{e}^{v}(N-\{j\}, x)$ for all $i, j \in N, i \neq j$.

Theorem 4.6. Let $v \in G^{n}$.

(i) $U(v) \cap \mathscr{K}^{*}(v) \subset\{\operatorname{ENSC}(v)\}$

(ii) $U(v) \cap \mathscr{K}^{*}(v)=\{\operatorname{ENSC}(v)\}$ iff $\operatorname{ENSC}(v) \in U(v)$.

Proof. (i) Suppose $U(v) \cap \mathscr{K}^{*}(v) \neq \varnothing$, say $x \in U(v) \cap$ $\mathscr{K}^{*}(v)$. By Proposition 4.1 and Lemma 4.5, we derive from $x \in U(v)$ that

$s_{i j}^{v}(x)=x_{j}-S C_{j}(v) \quad$ for all $i, j \in N, i \neq j, \quad$ while

$s_{i j}^{v}(x)=s_{j i}^{v}(x) \quad$ for all $i, j \in N, i \neq j$,

because of $x \in \mathscr{K}^{*}(v)$. From this, it follows immediately that

$x_{1}-S C_{1}(v)=x_{2}-S C_{2}(v)=\ldots=x_{n}-S C_{n}(v):=\alpha$.

Thus, we have that $x_{i}=S C_{i}(v)+\alpha$ for all $i \in N$. Together with $x(N)=v(N)$, this implies $\alpha=n^{-1} \mathrm{NSC}(v)$ and hence,

$x_{i}=S C_{i}(v)+n^{-1} \mathrm{NSC}(v)=\operatorname{ENSC}_{i}(v) \quad$ for all $i \in N$.

We conclude that $x=\operatorname{ENSC}(v)$ whenever $x \in U(v) \cap$ $\mathscr{K}^{*}(v)$.

(ii) The "only if" part is trivial. In order to prove the "if" part, suppose $z:=\operatorname{ENSC}(v) \in U(v)$. In view of part (i), it suffices to show $z \in \mathscr{K}^{*}(v)$. By Proposition 4.1 and Lemma 4.5 applied to the vector $z$, we deduce from $z \in U(v)$ that for all $i, j \in N, i \neq j$,

$s_{i j}^{v}(z)=z_{j}-S C_{j}(v)=\operatorname{ENSC}_{j}(v)-S C_{j}(v)=n^{-1} \mathrm{NSC}(v)$.

In particular, $s_{i j}^{v}(z)=s_{j i}^{v}(z)$ for all $i, j \in N, i \neq j$. So, $z \in \mathscr{K}^{*}(v)$ as was to be shown.
Theorem 4.7. Let $v \in G^{n}$.

(i) $\eta^{*}(v) \in U(v)$ iff $\operatorname{ENSC}(v) \in U(v)$

(ii) If $\operatorname{ENSC}(v) \in U(v)$, then $\eta^{*}(v)=\operatorname{ENSC}(v)$.

Proof. The "if" part of (i) as well as part (ii) are due to Proposition 4.1 and Theorem 3.1. To prove the "only if" part of (i), suppose $\eta^{*}(v) \in U(v)$. We always have $\eta^{*}(v) \in \mathscr{K}^{*}(v)$. From $\eta^{*}(v) \in U(v) \cap \mathscr{K}^{*}(v)$ and Theorem 4.6(i), we conclude that $\operatorname{ENSC}(v)=\eta^{*}(v) \in U(v)$. Therefore, (i) holds.

Finally, we treat the relationship between the set $U(v)$ and the part of the pre-imputation set that is bounded above by the smallest contributions of the single players to the nonempty coalitions. The formal definition of the relevant set $W(v)$, as presented in Funaki (1986), is as follows:

$$
\begin{aligned}
W(v):= & \left\{x \in I^{*}(v) \mid x_{i} \leq v(S \cup\{i\})-v(S)\right. \\
& \text { for all } i \in N \text { and all } S \subset N-\{i\}, S \neq \varnothing\} .
\end{aligned}
$$

Theorem 4.8. $W(v) \subset U(v)$ for all $v \in G^{n}$.

Proof. Let $x \in W(v)$ and let $S \subset N$ be such that $1 \leq|S| \leq n-2$. In order to prove $x \in U(v)$, we show that

$x(S) \leq v(N-\{j\})-v((N-\{j\})-S) \quad$ for all $j \in N-S$.

Let $j \in N-S$. Write $S=\left\{i_{1}, i_{2}, \ldots, i_{s}\right\}$ where $s:=|S|$ and put $\left[i_{0}\right]:=N-\{j\}, \quad\left[i_{k}\right]:=N-\left\{j, i_{1}, i_{2}, \ldots, i_{k}\right\}$ for all $1 \leq k \leq s$. Because $s \leq n-2$, we have that $\left[i_{k}\right] \neq \varnothing$ for all $1 \leq k \leq s$. Together with $x \in W(v)$, this implies that $x_{i_{k}} \leq v\left(\left[i_{k-1}\right]\right)-v\left(\left[i_{k}\right]\right)$ for all $1 \leq k \leq s$. Summing up these $s$ inequalities, we obtain

$$
\sum_{k=1}^{s} x_{i_{k}} \leq \sum_{k=1}^{s}\left(v\left(\left[i_{k-1}\right]\right)-v\left(\left[i_{k}\right]\right)\right)=v\left(\left[i_{0}\right]\right)-v\left(\left[i_{s}\right]\right) .
$$

Now it follows immediately that

$$
\begin{aligned}
x(S) & =\sum_{k=1}^{s} x_{i_{k}} \leq v\left(\left[i_{0}\right]\right)-v\left(\left[i_{s}\right]\right) \\
& =v(N-\{j\})-v((N-\{j\})-S)
\end{aligned}
$$

as was to be shown.

It is left to the reader to verify the emptiness of the set $W(w)$ for the game $w$ of Example 4.3, while the inclusion $W(v) \subset U(v)$ for the game $v$ of Example 4.2 is strict. The general inclusion enables us to present an alternative proof of Funaki's main results.

Corollary 4.9. (cf. Funaki 1986) Let $v \in G^{n}$.

(i) $W(v) \subset C(v)$

(ii) $W(v) \cap \mathscr{K}^{*}(v) \subset\{\operatorname{ENSC}(v)\}$ 
(iii) $W(v) \cap \mathscr{K}^{*}(v)=\{\operatorname{ENSC}(v)\}$ iff $\operatorname{ENSC}(v) \in W(v)$

(iv) $\eta^{*}(v) \in W(v)$ iff $\mathrm{ENSC}(v) \in W(v)$.

Proof. (i) The proof of the inclusion $W(v) \subset C(v)$ is similar to the proof of Theorem 4.8 .

(ii) From Theorem 4.8 and 4.6(i) respectively, we conclude

\section{$W(v) \cap \mathscr{K}^{*}(v) \subset U(v) \cap \mathscr{K}^{*}(v) \subset\{\operatorname{ENSC}(v)\}$}

(iii) The "only if" part is trivial. Further, the "if" part is a direct consequence of part (ii), Theorem 4.8 and 4.6(ii).

(iv) If $\operatorname{ENSC}(v) \in W(v)$, then $\operatorname{ENSC}(v) \in U(v)$ and hence, $\eta^{*}(v)=\operatorname{ENSC}(v) \in W(v)$ where the equality follows from Theorem 4.7(ii). The proof of the "only if" part is similar to the proof of part (i) of Theorem 4.7.

\section{Coincidence of and collinearity between solutions for glove games}

The previous two sections were devoted to the coincidence of the prenucleolus and the ENSC-solution. Henceforward, we mainly investigate the relationship between the ENSC-solution and the so-called Shapley value. To be exact, we consider two types of games for which the Shapley value can be obtained as a convex or affine combination of the ENSC-solution and the centre of the imputation set. Generally speaking, the centre of the $i$ mutation set (CIS-vector) of any $n$-person game $v$ is defined by

$\mathrm{CIS}_{i}(v):=v(\{i\})+n^{-1}\left[v(N)-\sum_{j \in N} v(\{j\})\right]$ for all $i \in N$.

For $n$-person games $v$ satisfying $v(\{i\})=0$ for all $i \in N$, the centre of the imputation set is characterized by the egalitarian distribution of the total worth $v(N)$ among the $n$ players in the game $v$, i.e., $\operatorname{CIS}_{i}(v)=n^{-1} v(N)$ for all $i \in N$.

In this section we treat a type of games which is formulated in terms of traders and gloves (cf. Shapley and Shubik 1969). The trader set $N$ is divided into two disjoint nonempty subsets $R$ and $L$ consisting of initial owners of right- and left-handed gloves. So, $N=R \cup L$ where $R \cap L=\varnothing$. Each trader possesses one glove and the worth of an assembled pair of gloves is one to whoever holds the pair. Due to cooperation between the traders of coalition $S$, the largest possible worth of the assembled pairs of gloves within $S$ is represented by

$v(S)=\min [|S \cap R|,|S \cap L|] \quad$ for all $S \subset N$.

Throughout the section we write $r=|R|$ and $l=|L|$. Obviously, the glove game $v$ of (5.1) satisfies $v(N)=\min (r, l)$ and $v(\{i\})=0$ for all $i \in N$. First of all, we elucidate the coincidence of the (pre)-nucleolus concept and the ENSC-method on the class of glove games. For that purpose, we study the validity of the conditions (3.3)(3.8) for a glove game.
Theorem 5.1. Let $v \in G^{n}$ be the glove game of (5.1).

(i) If $r=l$, then (3.8) does not hold.

(ii) If $r \neq l$, then Driessen's condition (3.7) as well as (3.8) hold, Legros' condition (3.5)-(3.6) does not hold, Funaki's condition (3.3) holds iff $r=1$ or $l=1$.

(iii) If $r=l$, then $\operatorname{ENSC}_{i}(v)=\frac{1}{2}$ for all $i \in N$.

If $r \neq l$, then $C(v)=\{\eta(v)\}=\{\operatorname{ENSC}(v)\}$. In addition, if $r<l$, then $\operatorname{ENSC}_{i}(v)=1$ for $i \in R$ and $\operatorname{ENSC}_{j}(v)=0$ for $j \in L$;

if $r>l$, then $\operatorname{ENSC}_{i}(v)=0$ for $i \in R$ and $\mathrm{ENSC}_{j}(v)=1$ for $j \in L$.

Proof. (i) Suppose $r=l$. Then we have $v(N)=r$, $v(N-\{i\})=r-1$ for all $i \in N$ and so, $S C_{i}(v)$ $=v(N)-v(N-\{i\})=1$ for all $i \in N$. Now it follows that $g^{v}(N)=r$ and $g^{v}(\{i, j\})=1$ for all $i \in R$, all $j \in L$. This yields that $n^{-1} g^{v}(N)=\frac{1}{2}>\frac{1}{3}=3^{-1} g^{v}(\{i, j\})$ for all $i \in R$, all $j \in L$. Therefore, the condition (3.8) does not hold. Moreover, the ENSC-solution is given by $\operatorname{ENSC}_{i}(v)=S C_{i}(v)+n^{-1} \operatorname{NSC}(v)=1-\frac{1}{2}=\frac{1}{2}$ for all $i \in N$.

(ii) \& (iii) It remains to consider the case $r \neq l$. Without loss of generality, suppose $r<l$ (otherwise, interchange the roles of the sets $R$ and $L)$. Then we have $v(N)=r$, $v(N-\{i\})=r-1$ for $i \in R, v(N-\{j\})=r$ for $j \in L$ and so, $S C_{i}(v)=1$ for $i \in R, S C_{j}(v)=0$ for $j \in L$. Now it follows that $g^{v}(S)=|S \cap R|-v(S) \geq 0$ for all $S \subset N$. In particular, $g^{v}(N)=0$. From this, it is obvious that the 1-convexity condition (3.7) as well as (3.8) hold. However, the monotonicity condition (3.5) for the gap function does not hold since $g^{v}(N)=0<1=g^{v}(\{i\})$ for all $i \in R$. In case $r \geq 2$, then $g^{v}(R)-g^{v}(R-\{i\})=1$ for all $i \in R$ and so, (3.3) does not hold. In case $r=1$, then $g^{v}(R)=1, g^{v}(S)=0$ for $S \neq R$ and as such, (3.3) holds. This proves the statement (ii). Because (3.8) holds and $g^{v}(N)=0$, we conclude from Proposition 3.2(ii) that $\eta(v)=\operatorname{ENSC}(v) \in C(v)$. Further, the core is at most a singleton because of $g^{v}(N)=0$. Therefore, $C(v)=\{\eta(v)\}=\{\operatorname{ENSC}(v)\}$. Moreover, we get that $\mathrm{ENSC}_{i}(v)=S C_{i}(v)$ for all $i \in N$. This proves the statement (iii).

Secondly, we direct the attention to the behaviour of the Shapley value on the class of glove games. The Shapley value of an arbitrary $n$-person game $v$ is usually seen as an efficient payoff vector which meets a certain expectation principle. That is, the expected payoff to any player $i \in N$ is derived from his marginal payments $v(S \cup\{i\})-v(S)$ for joining coalitions $S$ not containing player $i$ himself, and the probability distribution $\left\{n^{-1}\left(\begin{array}{c}n-1 \\ |S|\end{array}\right)^{-1} \mid S \subset N-\{i\}\right\}$ over the collection of all 
such coalitions. Formally, the Shapley value $\phi(v) \in \mathbb{R}^{n}$ of the game $v$ is given by its classical formula (cf. Shapley 1953): for all $i \in N$

$\phi_{i}(v):=\sum_{S \subset N-\{i\}} n^{-1}\left(\begin{array}{c}n-1 \\ |S|\end{array}\right)^{-1}[v(S \cup\{i\})-(v(S)]$.

It is well-known that the Shapley value is an efficient payoff vector, i.e., $\phi(v) \in I^{*}(v)$ for all $v \in G^{n}$. In addition, the Shapley value possesses the symmetry property because symmetrical players in a game receive the same payoff according to the Shapley value. Especially, the symmetry property of the Shapley value can be applied to the traders of $R$ and $L$ respectively in the glove game $v$ of (5.1). In fact, their Shapley value payoffs for the case $r \geq l$ are as follows (cf. Shapley and Shubik 1969):

$\phi_{i}(v)=\frac{1}{2}-\frac{r-l}{2 r} \sum_{k=0}^{l} \frac{r ! l !}{(r+k) !(l-k) !} \quad$ for $i \in R$,

$\phi_{j}(v)=\frac{1}{2}+\frac{r-l}{2 l} \sum_{k=1}^{l} \frac{r ! l !}{(r+k) !(l-k) !} \quad$ for $j \in L$.

The results for the case $r \leq l$ are obtainable by interchanging the roles of the sets $R$ and $L$ in the formula (5.3). If $r=l$, then it appears that the Shapley value, the nucleolus and the ENSC-solution coincide with the pay-

off vector $\left(\frac{1}{2}, \frac{1}{2}, \ldots, \frac{1}{2}\right) \in \mathbb{R}^{n}$. The following theorem describes the exact locus of the Shapley value of a glove game whenever $r>l$.

Theorem 5.2. Let $v \in G^{n}$ be the glove game of (5.1) where $r>l$. Then

$\phi(v)=\beta \operatorname{ENSC}(v)+(1-\beta) \operatorname{CIS}(v)$

where

$\beta:=\frac{r-l}{2 r}+\frac{(r+l)(r-l)}{2 r l} \sum_{k=1}^{l} \frac{r ! l !}{(r+k) !(l-k) !}$.

Proof. In view of Theorem 5.1(iii), the ENSC-solution for the case $r>l$ is given by $\operatorname{ENSC}_{i}(v)=0$ for $i \in R$ and $\operatorname{ENSC}_{j}(v)=1$ for $j \in L$. Further, $\operatorname{CIS}_{i}(v)=(r+l)^{-1} l$ for all $i \in N$. Consequently, we must establish that

$\phi_{i}(v)=(r+l)^{-1} l(1-\beta) \quad$ for $i \in R$,

$\phi_{j}(v)=\beta+(r+l)^{-1} l(1-\beta) \quad$ for $j \in L$.

For the sake of notation, we write

$\delta_{0}:=\sum_{k=0}^{l} \frac{r ! l !}{(r+k) !(l-k) !} \quad$ and

$\delta_{1}:=\sum_{k=1}^{l} \frac{r ! l !}{(r+k) !(l-k) !}$.
Then $\delta_{0}=1+\delta_{1} \quad$ as well as $\beta=(2 r)^{-1}(r-l)+$ $(2 r l)^{-1}(r+l)(r-l) \delta_{1}$. Due to the formula (5.3) for the Shapley value of $v$, we get

$\phi_{i}(v)=\frac{1}{2}-(2 r)^{-1}(r-l) \delta_{0} \quad$ for $i \in R$,

$\phi_{j}(v)=\frac{1}{2}+(2 l)^{-1}(r-l) \delta_{1} \quad$ for $j \in L$.

(i) First of all, we prove that $\phi_{l}(v)=(r+l)^{-1} l(1-\beta)$ for $i \in R$. Let $i \in R$. By straightforward calculations, we obtain

$\phi_{i}(v)=\frac{1}{2}-(2 r)^{-1}(r-l) \delta_{0}=(2 r)^{-1} l-(2 r)^{-1}(r-l) \delta_{1}$

and so,

$$
\begin{aligned}
l^{-1}(r+l) \phi_{i}(v) & =(2 r)^{-1}(r+l)-(2 r l)^{-1}(r+l)(r-l) \delta_{1} \\
& =1-\beta .
\end{aligned}
$$

(ii) Secondly, we prove that $\phi_{j}(v)-\phi_{i}(v)=\beta$ for all $i \in R$ and all $j \in L$. Let $i \in R$ and $j \in L$. Then we obtain

$\phi_{j}(v)-\phi_{i}(v)=(2 l)^{-1}(r-l) \delta_{1}+(2 r)^{-1}(r-l) \delta_{0}$

$=(2 l)^{-1}(r-l) \delta_{1}+(2 r)^{-1}(r-l)+(2 r)^{-1}(r-l) \delta_{1}$

$=(2 r)^{-1}(r-l)+(2 r l)^{-1}(r+l)(r-l) \delta_{1}=\beta$.

(iii) From the parts (i)-(ii), it follows immediately that $\phi_{j}(v)=\beta+\phi_{i}(v)=\beta+(r+l)^{-1} l(1-\beta) \quad$ for $j \in L, i \in R$.

This completes the proof.

Lemma 5.3. The real number $\beta$ of (5.4) satisfies $0<\beta<1$.

Proof. The strict inequality $\beta>0$ is a direct consequence of the formula (5.4) and the assumption $r>l$. Thus, it remains to show the strict inequality $\beta<1$. For the relevant glove game $v$ of (5.1), we have that $v(S \cup\{i\})-v(S) \in\{0,1\}$ for all $i \in N$ and all $S \subset N-\{i\}$. Together with the classical formula (5.2) for the Shapley value, this yields that

$0 \leq \phi_{i}(v) \leq \sum_{S \subset N-\{i\}} n^{-1}\left(\begin{array}{c}n-1 \\ |S|\end{array}\right)^{-1}=1 \quad$ for all $i \in N$

where the last equality holds because of the probability distribution. In point of fact, the strict inequality $\phi_{i}(v)>0$ holds for all $i \in N$ since $v(\{i, j\})-v(\{j\})=1$ whenever $i$ and $j$ are of a different type. We conclude that $0<\phi_{i}(v) \leq 1$ for all $i \in N$. Take $i \in R$ and $j \in L$. By part (ii) of the proof of Theorem 5.2, we have $\beta=\phi_{j}(v)-\phi_{i}(v)$. From this, $\phi_{i}(v)>0$ and $\phi_{j}(v) \leq 1$, it is now clear that $\beta<1$.

For any collection of one-point solution concepts which possess the symmetry property, the corresponding efficient solutions for the glove game $v$ of (5.1) lie on the 
same line because the traders of $R$ and $L$ respectively are symmetrical players in the glove game $v$. Theorem 5.2 and Lemma 5.3 state that the Shapley value of the glove game of (5.1) is a convex combination of the ENSC-solution and the centre of the imputation set with coefficients $\beta$ and $1-\beta$. If $r>l$, then the real number $\beta$ is defined by (5.4). In other words, the Shapley value of the glove game of (5.1) lies on the straight line segment with end points the ENSCsolution and the centre of the imputation set. The locus of the Shapley value on this line segment may coincide with the midpoint $\left(\right.$ e.g., $\beta=\frac{1}{2}$ in case $\left.r=2, l=1\right)$, it may lie near the centre of the imputation set $\left(\right.$ e.g., $\beta<\frac{1}{2}$ in case $r=3, l=2)$ or it may lie near the ENSC-solution (e.g., $\beta>\frac{1}{2}$ in case $\left.r=4, l=2\right)$. Further, it appears that the Shapley value almost coincides with the ENSCsolution whenever the number of left-handed gloves is fixed and the number of right-handed gloves approaches infinity. In the next section we establish a version of Theorem 5.2 for another type of games, the so-called $k$ coalitional $n$-person games.

\section{Collinearity between solutions for $\boldsymbol{K}$-coalitional games}

Example 6.1. Consider the 4-person glove game with two owners 1,2 of one right-handed glove, one owner 3 of two left-handed gloves and one owner 4 of one left-handed glove. The associated characteristic function $v$ is then given by

$v(N)=v(123)=2, \quad v(124)=v(134)=v(234)=1$,

$v(13)=v(14)=v(23)=v(24)=1$,

$v(S)=0$ otherwise.

Clearly, $S C_{i}(v)=1,1,1,0$ for $i=1,2,3,4$ respectively and $\operatorname{NSC}(v)=-1$. Thus, $\operatorname{ENSC}(v)=\frac{1}{4}(3,3,3,-1)$.

Because the nucleolus is equal to the unique core-element $(1,1,0,0)$, we have $\eta(v) \neq \mathrm{ENSC}(v)$. From the classical formula (5.2) we derive that the Shapley value $\phi(v)=$ $\frac{1}{12}(7,7,7,3)$. Observe that the Shapley value, the ENSC-solution and the centre of the imputation set lie on the same line in such a way that $\phi(v)=\frac{1}{3} \operatorname{ENSC}(v)$ $+\frac{2}{3} \operatorname{CIS}(v)$.

For the game of the above example, the Shapley value is the convex combination of the ENSC-solution and the centre of the imputation set with certain coefficients. The purpose of the section is to account for those coefficients by introducing a specific type of games, the so-called $k$-coalitional games. Especially, we describe the interrelationship between the Shapley value, the ENSC-solution and the CIS-vector on the class of $k$-coalitional $n$-person games.

By means of the integer $k$ satisfying $1 \leq k \leq n-1$, we divide the coalitions in an $n$-person game into three kinds:

- essential coalitions which contain precisely $k$ players, - small coalitions which consist of less than $k$ players,

- large coalitions which consist of more than $k$ players and different from the grand coalition $N$.

Definition 6.2. An $n$-person game $v$ is called a $k$-coalitional game if it satisfies the next two conditions.

(i) $v(S)=v(T) \quad$ for all $S, T \subset N$ with $|S|=|T|<k$.

(ii) there exist $\alpha_{T} \in \mathbb{R}$ for all $T \subset N,|T|=k$, and $\gamma_{k+1}$, $\gamma_{k+2}, \ldots, \gamma_{n-1} \in \mathbb{R}$ such that

$$
v(S)=\sum_{\substack{T \subset S,|T|=k}} \alpha_{T}+\gamma_{|S|} \quad \begin{aligned}
& \text { for all } S \subset N \\
& \text { with } k<|S|<n .
\end{aligned}
$$

Remark that nothing is required about the worth of any essential coalition in a $k$-coalitional game. Here $\alpha_{T} \in \mathbb{R}$ represents the maximal profit obtainable from the formation of the essential coalition $T$ within any large coalition. Concerning the condition (6.1), we note that the worth of any large coalition may be independent on the worths of essential coalitions. However, in case the profit $\alpha_{T}$ of any essential coalition $T$ for its formation within a large coalition happens to be the same as the worth $v(T)$ of the essential coalition in the game $v$, then the worth of any large coalition is completely determined by the worths of the essential subcoalitions and the coalition size dependent constant. For instance, the last situation applies to each unanimity $n$-person game $u_{R}, R \subset N, R \neq N, \varnothing$, which is given by

$$
\begin{array}{rlrl}
u_{R}(S):=1 & & \text { if } S \supset R \\
& =0 & & \text { otherwise. }
\end{array}
$$

Say $|R|=k$. Then the unanimity game $u_{R}$ is a $k$-coalitional game because the main condition (6.1) holds by choosing $\alpha_{T}=u_{R}(T)$ for all $T \subset N$ with $|T|=k$ and $\gamma_{k+1}=\gamma_{k+2}=\ldots,=\gamma_{n-1}=0$. Recall the well-known fact that the set $\left\{u_{R} \in G^{n} \mid R \subset N, R \neq \varnothing\right\}$ of all unanimity $n$-person games forms a basis of the class $G^{n}$.

The remainder of the section consists of three subsections which correspond to the cases $k=n-1, k=1$ and $2 \leq k \leq n-2$ respectively. Throughout each subsection we suppose that any $n$-person game $v$ is zero-normalized, i.e., $v(\{i\})=0$ for all $i \in N$. It is easily shown that the Shapley value $\phi$, the ENSC-solution and the CIS-vector possess the linearity property on the class of zeronormalized $n$-person games. That is, for all $n$-person games $v, w$ and all $\alpha, \beta \in \mathbb{R}$

$\psi(\alpha v+\beta w)=\alpha \psi(v)+\beta \psi(w)$ 
where $\psi \in\{\phi$, ENSC, CIS $\}$ and the $n$-person sum game $\alpha v+\beta w$ is given by $(\alpha v+\beta w)(S):=\alpha v(S)+\beta w(S)$ for all $S \subset N$. In each of the following three subsections we describe the exact locus of the Shapley value of a $k$-coalitional game in terms of the ENSC-solution and the CIS-vector.

Subsection 6.1. In case $k=n-1$, then the condition (6.1) is superfluous. So, an $n$-person game $v$ is an $(n-1)$-coalitional game if and only if the game is symmetric with respect to the coalition size up to $n-1$, i.e., $v(S)=v(T)$ for all $S, T \subset N$ with $|S|=|T|<n-1$. Evidently, any zero-normalized 3-person game can be seen as a 2-coalitional game. According to the next theorem, the Shapley value of an $(n-1)$-coalitional game is the convex combination of the ENSC-solution and the CIS-vector with coefficients $(n-1)^{-1}$ and $(n-1)^{-1}(n-2)$. Consequently, the Shapley value of any zero-normalized 3-person game is the midpoint of the straight line segment with end points the ENSC-solution and the CIS-vector. If $n>3$, the Shapley value of $(n-1)$-coalitional games lies near the CIS-vector and far off the ENSC-solution.

Theorem 6.3. If $v \in G^{n}$ is an (n-1)-coalitional game, then $\phi(v)=(n-1)^{-1} \operatorname{ENSC}(v)+(n-1)^{-1}(n-2) \operatorname{CIS}(v)$.

Proof. Let $v \in G^{n}$ be an $(n-1)$-coalitional game. Define the two $n$-person games $w$ and $z$ by

$$
\begin{array}{ll}
w(S):=v(S) & \text { if }|S|<n-1, \\
w(S):=0 & \text { if }|S| \geq n-1, \\
z(S):=0 & \text { if }|S|<n-1, \\
z(S):=v(S) & \text { if }|S| \geq n-1 .
\end{array}
$$

Then the equality $v(S)=w(S)+z(S)$ holds for all $S \subset N$. By applying the symmetry property of the Shapley value to the symmetric game $w$, we obtain that $\phi_{i}(w)=n^{-1} w(N)=0$ for all $i \in N$. Together with the linearity property of the Shapley value, this yields $\phi(v)=\phi(w+z)=\phi(w)+\phi(z)=\phi(z)$. From the classical formula (5.2) applied to the game $z$, we derive that for all $i \in N$

$$
\begin{aligned}
\phi_{i}(z)= & {[n(n-1)]^{-1} \sum_{j \in N-\{i\}} v(N-\{j\}) } \\
& +n^{-1}[v(N)-v(N-\{i\})] \\
= & {[n(n-1)]^{-1}\left[S C_{i}(v)-g^{v}(N)+(n-2) v(N)\right] } \\
& +n^{-1} S C_{i}(v) \\
= & (n-1)^{-1}\left[S C_{i}(v)-n^{-1} g^{v}(N)\right] \\
& +[n(n-1)]^{-1}(n-2) v(N) .
\end{aligned}
$$

From this and $\phi(v)=\phi(z)$, we conclude that for all $i \in N$

$$
\begin{aligned}
\phi_{i}(v)=\phi_{i}(z) & =(n-1)^{-1} \operatorname{ENSC}_{i}(v) \\
& +(n-1)^{-1}(n-2) \operatorname{CIS}_{i}(v) .
\end{aligned}
$$

Example 6.4. Consider the 4-person game $v$ given by

$v(N)=v(123)=v(124)=12, \quad v(S)=0$ otherwise.

Obviously, this 4-person game is a 3-coalitional game. Here $S C_{i}(v)=12,12,0,0$ for $i=1,2,3,4$ respectively and $\operatorname{NSC}(v)=-12$. Thus, $\operatorname{ENSC}(v)=(9,9,-3,-3)$ and as such, the ENSC-solution lies outside the imputation set. Further, the CIS-vector is given by $\operatorname{CIS}(v)=(3,3,3,3)$. Theorem 6.3 states that the Shapley value, the ENSC-solution and the CIS-vector lie on the same line $l$ in such a way that $\phi(v)=\frac{1}{3} \operatorname{ENSC}(v)$ $+\frac{2}{3} \operatorname{CIS}(v)$. As a result $\phi(v)=(5,5,1,1)$ which payoff vector is also obtainable from the classical formula (5.2) for the Shapley value. Besides, the core $C(v)$ is the straight line segment with end points $(12,0,0,0)$ and $(0,12,0,0)$. Because the players 1,2 are symmetrical in the game $v$, the nucleolus is determined as the midpoint of the core, i.e., $\eta(v)=(6,6,0,0)$. We observe that the nucleolus lies on the above line $l$ in such a way that $\phi(v)=\frac{2}{3} \eta(v)$ $+\frac{1}{3} \operatorname{CIS}(v)$.

Subsection 6.2. Now we direct the attention to the behaviour of solutions on the class of 1-coalitional games. For that purpose, we first treat three characterizations of a 1-coalitional game.

Proposition 6.5. Let $v \in G^{n}$. The following statements are equivalent.

(i) $v$ is a 1-coalitional game, i.e., (6.1) holds where $k=1$

(ii) there exist a unique $q \in I^{*}(v)$ and $\gamma_{2}, \gamma_{3}, \ldots, \gamma_{n-1} \in \mathbb{R}$ such that

$$
v(S)=q(S)+\gamma_{|S|} \quad \text { for all } S \subset N \text { with } 1<|S|<n
$$

(iii) $g^{v}(S)=g^{v}(T)$ for all $S, T \subset N$ with $1<|S|=|T|<n$.

Proof. We prove the implications (ii) $\Rightarrow$ (i) $\Rightarrow$ (iii) $\Rightarrow$ (ii). The implication (ii) $\Rightarrow$ (i) trivially holds.

(a) Suppose that (i) holds. Then there exist $\alpha=\left(\alpha_{1}, \alpha_{2}, \ldots, \alpha_{n}\right) \in \mathbb{R}^{n}$ and $\gamma_{2}, \gamma_{3}, \ldots, \gamma_{n-1} \in \mathbb{R}$ such that $v(S)=\alpha(S)+\gamma_{|S|}$ for all $S \subset N$ with $1<|S|<n$. It follows that for all $i \in N$

$S C_{i}(v)=v(N)-v(N-\{i\})=v(N)-\alpha(N)-\gamma_{n-1}+\alpha_{i}$ 
and hence,

$$
\begin{aligned}
g^{v}(S) & =\sum_{j \in S} S C_{j}(v)-v(S) \\
& =|S|\left[v(N)-\alpha(N)-\gamma_{n-1}\right]-\gamma_{|S|}
\end{aligned}
$$

for all $S \subset N$ with $1<|S|<n$. From this, it is clear that $g^{v}(S)=g^{v}(T)$ for all $S, T \subset N$ with $1<|S|=|T|<n$. So, (i) implies (iii).

(b) Suppose that (iii) holds. Choose the payoff vector $q \in I^{*}(v)$ and the real numbers $\gamma_{s}, 1<s<n$, by $q:=\operatorname{ENSC}(v)$ and $\gamma_{s}:=n^{-1} s g^{v}(N)-g^{v}(S)$ for all $S \subset N$ with $|S|=s$. The real numbers $\gamma_{s}$ are well-defined for $1<s<n$ because (iii) holds. It follows that for all $S \subset N$ with $1<|S|<n$

$$
\begin{aligned}
q(S)+\gamma_{|S|}= & \sum_{j \in S} \operatorname{ENSC}_{j}(v)+n^{-1}|S| g^{v}(N)-g^{v}(S) \\
= & \sum_{j \in S} S C_{j}(v)+n^{-1}|S| \operatorname{NSC}(v) \\
& +n^{-1}|S| g^{v}(N)-g^{v}(S)=v(S)
\end{aligned}
$$

This completes the proof of the existence part of statement (ii). In order to prove the uniqueness part for the preimputation $q$, suppose that (6.2) holds. By $q \in I^{*}(v)$ and (6.2), we obtain that for all $i \in N$

$$
\begin{aligned}
q_{i} & =v(N)-q(N-\{i\})=v(N)-v(N-\{i\})+\gamma_{n-1} \\
& =S C_{i}(v)+\gamma_{n-1} .
\end{aligned}
$$

Summing up these $n$ equalities, we deduce

$$
\begin{aligned}
& v(N)=q(N)=\sum_{j \in N} S C_{j}(v)+n \gamma_{n-1} \quad \text { or equivalently, } \\
& \gamma_{n-1}=n^{-1}\left[v(N)-\sum_{j \in N} S C_{j}(v)\right]=n^{-1} \mathrm{NSC}(v) .
\end{aligned}
$$

Consequently,

$q_{i}=S C_{i}(v)+\gamma_{n-1}=S C_{i}(v)+n^{-1} \mathrm{NSC}(v)=\operatorname{ENSC}_{i}(v)$ for all $i \in N$.

We conclude that the pre-imputation $q$ coincides with the ENSC-solution. This completes the proof of the uniqueness part of statement (ii). So, (iii) implies (ii).

The unique pre-imputation $q$ to which Proposition 6.5(ii) refers is called the quota vector of the 1-coalitional game $v$. As a matter of fact, each player $i \in N$ in a 1-coalitional game has a quota $q_{i} \in \mathbb{R}$ potentially and the players can get their quotas solely by the formation of multiperson coalitions. In addition to the sum of the members' quotas, each multiperson coalition can gain or lose an additional amount which varies with the size of the coalition involved. Due to the above characterization, a 1-coali- tional game is also called a quasi quota game. Recall that the quota vector coincides with the ENSC-solution.

Another characterization states that a quasi quota game is completely determined by the symmetry of the corresponding gap function on the multiperson coalitions. As a direct consequence of the last characterization, any 3-person game can be seen as a quasi quota game. The next theorem expresses that the Shapley value of a quasi quota $n$-person game is the convex combination of the ENSC-solution and the CIS-vector with coefficients $(n-1)^{-1}(n-2)$ and $(n-1)^{-1}$. In contradistinction to the locus of the Shapley value of $(n-1)$-coalitional games, the Shapley value of quasi quota $n$-person games where $n>3$ lies near the ENSC-solution and far off the CIS-vector.

Theorem 6.6. If $v \in G^{n}$ is a quasi quota game, then

$\phi(v)=(n-1)^{-1}(n-2) \operatorname{ENSC}(v)+(n-1)^{-1} \operatorname{CIS}(v)$.

Proof. Let $v \in G^{n}$ be a quasi quota game with quota vector $q$ and coalition size dependent constants $\gamma_{s}, 1 \leq s \leq n$, where $\gamma_{1}=\gamma_{n}=0$. Define the two $n$-person games $w$ and $z$ by

$w(S):=\gamma_{s} \quad$ for all $S \subset N, S \neq \varnothing$, with $|S|=s$,

$z(\{i\}):=-q_{i} \quad$ for all $i \in N$,

$z(S):=0 \quad$ otherwise.

Then the equality $v(S)=q(S)+w(S)+z(S)$ holds for all $S \subset N$. Together with the linearity property of the Shapley value on $G^{n}$, this implies $\phi(v)=q+\phi(w)+\phi(z)$. Furthermore, the symmetry property of the Shapley value applied to the symmetric game $w$ yields that $\phi_{i}(w)=n^{-1} w(N)=0$ for all $i \in N$. From (5.2) applied to the game $z$, we derive that for all $i \in N$

$\phi_{i}(z)=n^{-1} z(\{i\})-[n(n-1)]^{-1} \sum_{j \in N-\{i\}} z(\{j\})$

and hence,

$(n-1) \phi_{i}(z)=n^{-1} q(N-\{i\})-n^{-1}(n-1) q_{i}=n^{-1} q(N)-q_{i}$.

Since the quota vector $q=\operatorname{ENSC}(v)$, we conclude that for all $i \in N$

$$
\begin{aligned}
(n-1) \phi_{i}(v) & =(n-1) q_{i}+(n-1) \phi_{i}(z)=(n-1) q_{i} \\
& +n^{-1} q(N)-q_{i}=(n-2) q_{i}+n^{-1} v(N) \\
& =(n-2) \mathrm{ENSC}_{i}(v)+\mathrm{CIS}_{i}(v) .
\end{aligned}
$$

Because any zero-normalized 3-person game can be regarded as both a quasi quota game and a 2-coalitional game, we obtain that the two Theorems 6.3 and 6.6 are identical for zero-normalized 3-person games. Finally, we investigate the coincidence of the ENSC-method and the nucleolus concept on the class of quasi quota $n$-person games. 
Corollary 6.7. Let $v \in G^{n}$ be a quasi quota game with quota vector $q$ and coalition size dependent constants $\gamma_{s}, 1<s<n$.

(i) The condition (3.8) holds iff

$$
\begin{aligned}
& \gamma_{s} \leq \gamma_{n-1} \quad \text { for all } 1<s<n \quad \text { and } \\
& -q_{i} \leq \gamma_{n-1} \quad \text { for all } i \in N .
\end{aligned}
$$

(ii) If (6.3) holds and $\gamma_{n-1} \leq 0$, then $\eta(v)=\operatorname{ENSC}(v)$ and $\phi(v)=(n-1)^{-1}(n-2) \eta(v)+(n-1)^{-1} \operatorname{CIS}(v)$.

Proof. It was already shown in the second part of (b) in the proof of Proposition 6.5 that $S C_{i}(v)=q_{i}-\gamma_{n-1}$ for all $i \in N$. It follows that for all $S \subset N$

$g^{v}(S)=\sum_{j \in S} S C_{j}(v)-v(S)=q(S)-v(S)-|S| \gamma_{n-1}$. So, $g^{v}(N)=-n \gamma_{n-1}, \quad g^{v}(\{i\})=q_{i}-\gamma_{n-1} \quad$ for all $i \in N$, $g^{v}(S)=-s \gamma_{n-1}-\gamma_{s}$ for all $S \subset N$ with $|S|=s, 1<s<n$.

In view of this, the equivalence mentioned in statement (i) is obtained by straightforward calculations. Moreover, the statement (ii) is a direct consequence of part (i), Proposition 3.2(ii) and Theorem 6.6.

Concerning quasi quota $n$-person games, the condition (6.3) together with $\gamma_{n-1} \leq 0$ are sufficient but not necessary for the coincidence of the ENSC-method and the nucleolus concept. The nonnecessity is illustrated by the following example.

Example 6.8. Consider the zero-normalized 4-person game $v$ given by

$v(N)=12, \quad v(123)=v(124)=6, \quad v(134)=v(234)=0$,

$v(12)=12, \quad v(13)=v(14)=v(23)=v(24)=6, \quad v(34)=0$.

This 4-person game is a quasi quota game with quota vector $q=(6,6,0,0)$ and coalition size dependent constants $\gamma_{2}=0, \gamma_{3}=-6$. Thus, the condition (6.3) does not hold, but nevertheless $\operatorname{ENSC}(v)=\eta(v)=(6,6,0,0)$. Here the ENSC-solution is derived from the quota vector $q$, while the nucleolus is determined as the unique core-element. Note that the Shapley value can be calculated with the aid of Theorem 6.6 and as a result, we get $\phi(v)=\frac{2}{3} \operatorname{ENSC}(v)+\frac{1}{3} \operatorname{CIS}(v)=(5,5,1,1)$.

The 4-person game $v$ of Example 6.4 is not a quasi quota game because the corresponding gap function is not symmetric with respect to the multiperson coalitions, e.g., $g^{v}(12)=24, g^{v}(34)=0$. It is left to the reader to verify that the 4-person glove game $v$ of Example 6.1 is neither a quasi quota game nor a 3-coalitional game.

Subsection 6.3. The main object is to describe the exact locus of the Shapley value on a subclass of $k$-coalitional $n$-person games where $2 \leq k \leq n-2$. In order to formu- late the main theorem, we first fix some notation. The set of all essential coalitions is denoted by $\Gamma_{k}$, i.e.,

$\Gamma_{k}:=\{T|T \subset N| T \mid,=k\}$

Consider any $n$-person game $v$ and any real numbers $\alpha_{T}$, $T \in \Gamma_{h}$. For any player $i \in N$, we define the set $\Gamma_{k}^{i}$ of coalitions and the real numbers $v^{i}, \alpha^{i}$ by

$\Gamma_{k}^{i}:=\left\{T \mid T \in \Gamma_{k}, i \in T\right\}$,

$v^{i}:=\sum_{T \in \Gamma_{k}^{i}} v(T), \quad \alpha^{i}:=\sum_{T \in \Gamma_{k}^{i}} \alpha_{T}$.

Here the real number $v^{i}$ ( $\alpha^{i}$ respectively) represents the total amount what player $i$ can attain by acting as a member of essential coalitions with respect to the formation of the essential coalitions within the game $v$ itself (within large coalitions). The real numbers $\bar{v}$ and $\bar{\alpha}$ are determined by averaging, i.e.,

$\bar{v}:=n^{-1} \sum_{j \in N} v^{j} \quad$ and $\quad \vec{\alpha}:=n^{-1} \sum_{j \in N} \alpha^{j}$.

According to the main theorem, the Shapley value of a $k$-coalitional $n$-person game where $2 \leq k \leq n-2$ is an affine combination of the ENSC-solution and the CISvector, on the understanding that the quotient of the two deviations $\bar{v}-v^{i}$ and $\bar{\alpha}-\alpha^{i}$ is the same for all players $i \in N$.

Theorem 6.9. Let $v \in G^{n}$ be a $k$-coalitional game such that (6.1) holds and $2 \leq k \leq n-2$. If $\left(\bar{\alpha}-\alpha^{i}\right)^{-1}\left(\bar{v}-v^{i}\right)=c$ for all $i \in N$ and a certain constant $c \in \mathbb{R}$, then

$\phi(v)=\varrho \operatorname{ENSC}(v)+(1-\varrho) \operatorname{CIS}(v)$

where

$\varrho:=\left[k\left(\begin{array}{c}n-1 \\ k\end{array}\right)\right]^{-1}\left[\left(\begin{array}{c}n-1 \\ k\end{array}\right)-1+c\right]$.

The proof of Theorem 6.9 will be given at the end of this subsection. It may happen that the maximal profit $\alpha_{T}$, obtainable from the formation of the essential coalition $T$ within any large coalition, is equal to the original worth $v(T)$ in the game $v$, i.e., $\alpha_{T}=v(T)$ for all $T \subset N$ with $|T|=k$. Under these circumstances, it follows immediately that $v^{i}=\alpha^{i}$ for all $i \in N, \bar{v}=\bar{\alpha}$ and hence, Theorem 6.9 reduces to the next corollary.

Corollary 6.10. Let $v \in G^{n}, 2 \leq k \leq n-2$, and suppose there exist $\gamma_{k+1}, \gamma_{k+2}, \ldots, \gamma_{n-1} \in \mathbb{R}$ such that

(i) $\quad v(S)=v(T) \quad$ for all $S, T \subset N$ with $|S|=|T|<k$.

(ii) $v(S)=\sum_{\substack{T \subset S,|T|=k}} v(T)+\gamma_{|S|} \quad \begin{aligned} & \text { for all } S \subset N \\ & \text { with } k<|S|<n .\end{aligned}$ 
Then $\phi(v)=k^{-1} \operatorname{ENSC}(v)+k^{-1}(k-1) \operatorname{CIS}(v)$.

It is left to the reader to verify that the 4-person glove game $v$ of Example 6.1 is a 2-coalitional game with respect to the profits $\alpha_{12}=\alpha_{13}=\alpha_{14}=\frac{1}{2}, \alpha_{23}=1, \alpha_{24}=\alpha_{34}=0$ and the coalition size dependent constant $\gamma_{3}=0$. Notice that the profits are not uniquely determined. Straightforward calculations yield that $v^{i}=2$ for all $i \in N, \bar{v} \bar{\tau} 2$ and so, $\bar{v}-v^{i}=0$ for all $i \in N$. From this, we conclude that Theorem 6.9 applies to the 2-coalitional 4-person glove game $v$ and indeed, the Shapley value is the convex combination of the ENSC-solution and the CISvector with coefficients $\frac{1}{3}$ and $\frac{2}{3}$ as was shown in Example 6.1.

In case the constant $c$ to which Theorem 6.9 refers is equal to zero (cf. Example 6.1), then the real number $\varrho$ of (6.4) satisfies $0<\varrho<\frac{1}{2}$ and therefore, the Shapley value is even a convex combination of the ENSC-solution and the CIS-vector. The next example illustrates that the affine combination to which Theorem 6.9 refers is not necessarily a convex combination.

Example 6.11. Consider the 4-person game $v$ given by

$$
\begin{aligned}
& v(N)=v(123)=v(124)=v(134)=12, \\
& v(12)=v(13)=v(14)=6 \delta, \quad v(S)=0 \quad \text { otherwise, }
\end{aligned}
$$

where $\delta$ is an arbitrary real number. It is straightforward to check that this 4-person game is a 2-coalitional game with respect to the profits $\alpha_{12}=\alpha_{13}=\alpha_{14}=6$, $\alpha_{23}=\alpha_{24}=\alpha_{34}=0$ and the constant $\gamma_{3}=0$. Further, it appears that $\bar{v}-v^{i}=\delta\left(\bar{\alpha}-\alpha^{i}\right)$ for all $i \in N$. By applying Theorem 6.9, the Shapley value is an affine combination of the ENSC-solution $(12,0,0,0)$ and the CISvector $(3,3,3,3)$ in such a way that $\phi(v)=$ $\varrho \operatorname{ENSC}(v)+(1-\varrho) \operatorname{CIS}(v)$ where $\varrho=\frac{1}{6}(2+\delta)$. Evidently, $\phi(v)$ lies on the straight line segment with end points $\operatorname{ENSC}(v)$ and CIS $(v)$ if and only if $-2 \leq \delta \leq 4$.

Example 6.12. Consider the 4-person glove game with two owners 1, 2 of two right-handed gloves, one owner 3 of one left-handed glove and one owner 4 of two left-handed gloves. The associated characteristic function $v$ is then given by

$$
\begin{aligned}
& v(N)=3, \quad v(124)=v(134)=v(234)=2, \quad v(123)=1, \\
& v(13)=v(23)=1, \quad v(14)=v(24)=2, \\
& v(S)=0 \quad \text { otherwise. }
\end{aligned}
$$

This 4-person game is a 2-coalitional game with respect to the profits $\alpha_{12}=0, \alpha_{13}=\alpha_{23}=\alpha_{34}=\frac{1}{2}, \alpha_{14}=\alpha_{24}=1$ and the constant $\gamma_{3}=0$. Straightforward calculations yield the solutions $\operatorname{CIS}(v)=\frac{1}{4}(3,3,3,3), \operatorname{ENSC}(v)=$ $\frac{1}{2}(1,1,1,3)$ and $\phi(v)=\frac{1}{6}(4,4,3,7)$. We observe that these three solutions of the 2-coalitional 4-person game $v$ are not on the same line. The reason for this is that there exist no profits $\alpha_{T}, T \subset N,|T|=2$, satisfying (6.1) as well as $\bar{\alpha}-\alpha^{3}=\alpha^{4}-\bar{\alpha}$. The reader is invited to verify this fact. We remark that if owner 1 possesses only one instead of two right-handed gloves, then the Shapley value of the induced 2-coalitional 4-person glove game is the midpoint of the straight line segment with end points the ENSCsolution and the CIS-vector.

Proof of Theorem 6.9. Let $v \in G^{n}$ be a $k$-coalitional game such that (6.1) holds and $2 \leq k \leq n-2$. Define the two $n$ person games $w$ and $z$ by

$$
\begin{array}{rlrl}
w(S): & =v(S) & & \text { if }|S|<k \\
& =\gamma_{|S|} & & \text { if } k<|S|<n \\
& =0 & \text { if } S \in \Gamma_{k} \text { or } S=N, \\
z(S): & =v(S)-\alpha_{S} & & \text { for all } S \in \Gamma_{k} \\
& =v(N)-\sum_{T \in \Gamma_{k}} \alpha_{T} & & \text { for } S=N \\
& =0 & & \text { for all } S \neq N, S \notin \Gamma_{k} .
\end{array}
$$

Then the equality $v(S)=\sum_{T \in \Gamma_{k}} \alpha_{T} u_{T}(S)+w(S)+z(S)$ holds for all $S \subset N$. Shortly, $v=\sum_{T \in \Gamma_{k}} \alpha_{T} u_{T}+w+z$ where $u_{T}$ is the unanimity $n$-person game with respect to the essential coalition $T$.

(i) The game $w$ is zero-normalized as well as symmetric. Therefore, we obtain that $\phi_{i}(w)=\mathrm{CIS}_{i}(w)=n^{-1} w(N)=0$ for all $i \in N$. Further, we have that $S C_{i}(w)=w(N)-$ $w(N-\{i\})=-\gamma_{n-1}$ for all $i \in N$ and so, $N S C(w)=n \gamma_{n-1}$. It follows that $\operatorname{ENSC}_{i}(w)=S C_{i}(w)+n^{-1} \mathrm{NSC}(w)=0$ for all $i \in N$. As a result, $\phi_{i}(w)=\operatorname{CIS}_{i}(w)=\operatorname{ENSC}_{i}(w)=0$ for all $i \in N$.

(ii) Let $T \in \Gamma_{k}$. For all $i \in N$ and all $S \subset N-\{i\}$, we have

$$
\begin{aligned}
u_{T}(S \cup\{i\})-u_{T}(S) & =1 & & \text { if } i \in T, T-\{i\} \subset S \\
& =0 & & \text { otherwise. }
\end{aligned}
$$

Together with (5.2), this implies that $\phi_{i}\left(u_{T}\right)=0$ for all $i \in N-T$. Because the members of $T$ are symmetrical in the game $u_{T}$ and the Shapley value is always a preimputation, it follows that $\phi_{i}\left(u_{T}\right)=k^{-1}$ for all $i \in T$. Since $|T|=k \geq 2$, the game $u_{T}$ is zero-normalized and thus, $\operatorname{CIS}_{i}\left(u_{T}\right)=n^{-1}$ for all $i \in N$. In order to determine the ENSC-solution, we first note that $S C_{i}\left(u_{T}\right)=1$ for all $i \in T$, $S C_{i}\left(u_{T}\right)=0$ for all $i \in N-T$ and $\operatorname{NSC}\left(u_{T}\right)=1-k$. These calculations yield 


$$
\begin{aligned}
\operatorname{ENSC}_{i}\left(u_{T}\right) & =n^{-1}(n+1-k) & & \text { for all } i \in T \\
& =n^{-1}(1-k) & & \text { for all } i \in N-T .
\end{aligned}
$$

(iii) Because of $2 \leq k \leq n-2$, we have that $z$ ( $\{i\})$ $=0=z(N-\{i\})$ for all $i \in N$. From this we derive that $\operatorname{ENSC}_{i}(z)=\operatorname{CIS}_{i}(z)=n^{-1} z(N)$ for all $i \in N$. From (5.2) applied to the game $z$, we deduce that for all $i \in N$

$$
\begin{aligned}
n ! \phi_{i}(z)= & (n-1) ! z(N) \\
& +(k-1) !(n-k) ! \sum_{T \in \Gamma_{k}^{i}}\left[v(T)-\alpha_{T}\right] \\
& -k !(n-k-1) ! \sum_{T \in V_{k}^{i}}\left[v(T)-\alpha_{T}\right]
\end{aligned}
$$

where $V_{k}^{i}:=\left\{T \mid T \in \Gamma_{k}, i \notin T\right\}$. Due to a combinatorial argument, we have both

$$
\begin{aligned}
& \sum_{T \in \Gamma_{k}} v(T)=k^{-1} \sum_{j \in N} v^{j}=k^{-1} n \bar{v} \quad \text { and } \\
& \sum_{T \in \Gamma_{k}} \alpha_{T}=k^{-1} \sum_{j \in N} \alpha^{j}=k^{-1} n \bar{\alpha} .
\end{aligned}
$$

As a consequence, we obtain that for all $i \in N$

$$
\begin{aligned}
& n ! \phi_{i}(z)=(n-1) ! z(N)+(k-1) !(n-k) !\left(v^{i}-\alpha^{i}\right) \\
& -k !(n-k-1) !\left(k^{-1} n \bar{v}-v^{i}-k^{-1} n \bar{\alpha}+\alpha^{i}\right) \quad \text { and so, } \\
& \phi_{i}(z)=n^{-1} z(N)+\left[k\left(\begin{array}{c}
n-1 \\
k
\end{array}\right)\right]^{-1}\left[\bar{\alpha}-\bar{v}+v^{i}-\alpha^{i}\right] .
\end{aligned}
$$

(iv) The three solutions for the game $v$ are determined by combining the obtained results of the parts (i)-(iii) on the basis of the linearity property of the three solutions on the class of zero-normalized $n$-person games. In point of fact, we conclude that for all $i \in N$

$$
\begin{aligned}
\operatorname{CIS}_{i}(v)= & \sum_{T \in \Gamma_{h}} n^{-1} \alpha_{T}+n^{-1} z(N)=k^{-1} \bar{\alpha}+n^{-1} z(N), \\
\operatorname{ENSC}_{i}(v)= & \sum_{T \in \Gamma_{k}^{i}} n^{-1} \alpha_{T}(n+1-k) \\
& +\sum_{T \in V_{k}^{i}} n^{-1} \alpha_{T}(1-k)+n^{-1} z(N) \\
= & \alpha^{i}+k^{-1}(1-k) \bar{\alpha}+n^{-1} z(N), \\
\phi_{i}(v)= & k^{-1} \alpha^{i}+\left[k\left(\begin{array}{c}
n-1 \\
k
\end{array}\right)\right]^{-1}\left[\bar{\alpha}-\bar{v}+v^{i}-\alpha^{i}\right] \\
& +n^{-1} z(N) .
\end{aligned}
$$

(v) From now we suppose that $\left(\bar{\alpha}-\alpha^{i}\right)^{-1}\left(\bar{v}-v^{i}\right)=c$ for all $i \in N$ and a certain constant $c \in \mathbb{R}$. Let $i \in N$ and let the real number $\varrho$ be defined by (6.4). It remains to verify the equality

$$
\phi_{i}(v)=\operatorname{CIS}_{i}(v)+\varrho\left[\operatorname{ENSC}_{i}(v)-\operatorname{CIS}_{i}(v)\right]
$$

or equivalently,

$$
\begin{aligned}
& k^{-1}\left(\alpha^{i}-\bar{\alpha}\right)+\left[k\left(\begin{array}{c}
n-1 \\
k
\end{array}\right)\right]^{-1}\left[\bar{\alpha}-\alpha^{i}+v^{i}-\bar{v}\right] \\
& =\varrho\left[\alpha^{i}-\bar{\alpha}\right]
\end{aligned}
$$

or equivalently,

$\varrho=k^{-1}+\left[k\left(\begin{array}{c}n-1 \\ k\end{array}\right)\right]^{-1}[-1+c]$.

Clearly, the last equality holds because of the definition of the real number $\varrho$.

Theorem 6.9 deals with $k$-coalitional $n$-person games where $2 \leq k \leq n-2$, but the case $k=1$ can also be added by presenting a slightly adapted proof (involving the CISvector for the unanimity games and the game $z$ ). As a matter of fact, $k=1$ implies $v^{i}=v(\{i\})=0$ for all $i \in N$, $\bar{v}=0, \varrho=(n-1)^{-1}(n-2)$. So, actually, Theorem 6.9 has been proved for the case $k=1$ (namely Theorem 6.6).

\section{Concluding remarks}

Remark 7.1. Notice the resemblance of the CIS-vector to the ENSC-solution whenever the principle of a minimal payoff (i.e., the worth $v(\{i\})$ of any single player i) is replaced by the principle of a maximal payoff (i.e., the separable contribution $S C_{i}(v)$ of any single player i). In point of fact, the relevant resemblance can be supported with the aid of the notion of the dual game. Given an $n$-person game $v$, its dual game $v * \in G^{n}$ is defined by $v^{*}(S):=v(N)-v(N-S)$ for all $S \subset N$. Here $v^{*}(S)$ represents the contribution of coalition $S$ with respect to the formation of the grand coalition $N$ in the game $v$. It is easily shown that the CIS-vector of the game $v$ agrees with the ENSC-solution of the dual game $v^{*}$. That is, $\operatorname{CIS}(v)=\operatorname{ENSC}\left(v^{*}\right)$ for all $v \in G^{n}$.

Remark 7.2. The dimension of the linear space $G_{0}^{n}$ of all zero-normalized $n$-person games is equal to $2^{n}-n-1$. The class of $k$-coalitional $n$-person games is a linear space within $G_{0}^{n}$ and its dimension equals

$$
2 n-2 \quad \text { for } k=1 \text { or } k=n-1 \text {, }
$$$$
2\left(\begin{array}{l}
n \\
k
\end{array}\right)+n-2 \text { for } 2 \leq k \leq n-2 \text {. }
$$

Acknowledgement. The authors are grateful to two anonymous referees for their helpful comments which led to improvements in the presentation of the paper.

\section{References}

Driessen TSH (1985) Properties of 1-convex $n$-person games. OR Spektrum 7:19-26

Driessen TSH (1988) Cooperative games, solutions and applications. Kluwer Academic Publishers, Dordrecht, The Netherlands 
Driessen TSH, Tijs SH (1983) The $\tau$-value, the nucleolus and the core for a subclass of games. Methods Operations Res 46:395406

Funaki Y (1986) Upper and lower bounds of the kernel and nucleolus. Int J Game Theory 15:121-129

Legros $P$ (1986) Allocating joint costs by means of the nucleolus. Int J Game Theory 15:109-119

Schmeidler D (1969) The nucleolus of a characteristic function game. SIAM J Appl Math 17:1163-1170

Shapley LS (1953) A value for $n$-person games. In: Kuhn H, Tucker AW (eds) Contributions to the theory of games II. Princeton
University Press, Princeton, New Jersey, pp 307-317

Shapley LS, Shubik M (1969) Pure competition, coalitional power, and fair division. Int Econ Rev 10:337-362

Suzuki M, Nakamura K (1976) Social systems; a game theoretic approach (Japanese). Kyoritsu Shuppan, Tokyo

Suzuki M, Nakayama M (1976) The cost assignment of the cooperative water resource development: a game theoretical approach. Manage Sci 22:1081-1086

Young HP, Okada N, Hashimoto T (1982) Cost allocation in water resources development. Water Resources Res 18:463-475 